BULLETIN Bulletin hispanique

HISPANIQUE Université Michel de Montaigne Bordeaux

118-1 | 2016

La Guerre Civile espagnole aujourd'hui (1936-2016)

\title{
Les compagnies de travailleurs étrangers (CTE) en France à la fin de la Troisième République
}

\section{Vincent Parello}

\section{(2) OpenEdition}

\section{Journals}

\section{Édition électronique}

URL : http://journals.openedition.org/bulletinhispanique/4328

DOI : 10.4000/bulletinhispanique.4328

ISSN : 1775-3821

\section{Éditeur}

Presses universitaires de Bordeaux

\section{Édition imprimée}

Date de publication : 15 juillet 2016

Pagination : 233-250

ISBN : 979-10-300-0058-0

ISSN : 0007-4640

\section{Référence électronique}

Vincent Parello, «Les compagnies de travailleurs étrangers (CTE) en France à la fin de la Troisième République », Bulletin hispanique [En ligne], 118-1 | 2016, mis en ligne le 15 juillet 2019, consulté le 06 septembre 2019. URL : http://journals.openedition.org/bulletinhispanique/4328 ; DOI : 10.4000/ bulletinhispanique.4328 


\title{
Les compagnies de travailleurs étrangers (CTE) en France à la fin de la Troisième République
}

\author{
VinCENT PARELlo \\ Université Bordeaux Montaigne
}

Cet article analyse la création des compagnies de travailleurs étrangers (CTE) en France à la fin de la Troisième République. Par un décret-loi du 12 avril 1939, environ 55000 réfugiés espagnols furent contraints au travail obligatoire, principalement au service des armées, dans l'agriculture et dans l'industrie de l'armement.

Mots-clés: compagnies de travailleurs étrangers, CTE, Troisième République, réfugiés espagnols, travail obligatoire.

Este artículo analiza la creación de las compañías de trabajadores extranjeros (CTE) en Francia a finales de la Tercera República. Por un dereto-ley del 12 de abril de 1939, unos 55000 refugiados españoles fueron forzados a trabajar, principalmente en los ejércitos, en la agricultura y en las industrias de armamento.

Palabras claves: compañías de trabajadores extranjeros, CTE, Tercera República, refugiados españoles, trabajo obligatorio.

This article studies the creation of the Companies of Foreign Workers (CTE) in France towards the end of the Third Republic. Through a decree of 12th April 1939, about 55000 Spanish refugees were forced to work, mostly for the Army, in the agricultural sector and for the arms industry.

Keywords: the Compagnies of Foreign Workers, CTE, Third republic, Spanish refugees, compulsory work.

\footnotetext{
A la fin de la Troisième République, et avec l'entrée en guerre de la France contre l'Allemagne, en septembre 1939, les conditions de vie des réfugiés espagnols allèrent de Charybde en Scylla. En gros, trois possibilités s'offraient à eux selon leur condition: le rapatriement en Espagne, le départ vers un nouveau
} 
pays d'accueil (Amérique Latine et URSS principalement), ou l'installation en France. Les enfants dont les parents résidaient en Espagne, les orphelins, les femmes et leur descendance qui ne pouvaient justifier que leur soutien naturel se trouvait sur place, les malades et les invalides qui s'étaient réfugiés en France " non pour fuir des représailles politiques mais pour se soustraire aux dangers de la Guerre civile ", furent vivement encouragés à quitter leur pays d'accueil dans les plus brefs délais. Seuls étaient autorisés à demeurer dans l'Hexagone, les réfugiés valides susceptibles d'occuper un emploi salarié, les invalides, les blessés et les « incurables " qui ne pouvaient retourner en Espagne sans risquer de graves représailles, les familles dont le chef ou l'un des membres avait été pourvu d'un emploi salarié, appartenait à une compagnie de travailleurs étrangers (CTE), était engagé dans une formation combattante, ou se trouvait interné à titre administratif dans un camp ${ }^{1}$.

\section{LE CADRE LÉGAL DES COMPAGNIES DE TRAVAILLEURS ÉTRANGERS}

De toute évidence, il existe une solution de continuité entre l'exploitation de la main-d'œuvre indigène dans les colonies françaises pendant l'entre-deuxguerres, la création des CTE à la fin de la Troisième République, et la mise en place du système des groupes de travailleurs étrangers (GTE) sous le régime de Vichy ${ }^{2}$. Dans la plus pure tradition coloniale et capitaliste, il s'agissait de faire face à la crise économique, d'exercer un contrôle idéologique sur certaines minorités et de réaliser une plus-value à partir de la main-d'œuvre du travailleur.

En 1930, l'Organisation internationale du Travail (OIT) adopta une convention qui invitait l'ensemble des pays signataires à "supprimer l'emploi du travail forcé ou obligatoire ", considéré comme une atteinte grave aux droits de l'homme et une restriction de sa liberté ${ }^{3}$. Imposé par l'État ou des agents privés à des fins d'exploitation économique, le travail forcé pouvait revêtir de multiples facettes, telles que l'esclavage organisé, la participation obligatoire à des travaux publics, l'emploi dans l'agriculture, la servitude pour dettes, le travail domestique ou les travaux pénitentiaires ${ }^{4}$.

Il apparaît clairement que cette convention tentait d'imposer et de promouvoir le travail libre dans l'ensemble des pays du monde occidental. Toutefois, il n'est pas inutile de souligner qu'elle se heurta à l'hostilité de la France qui, tout

1. Archives Départementales de l'Hérault $(\mathrm{ADH}), 2 \mathrm{~W} 602$. Circulaire du ministre de l'Intérieur aux préfets (19/09/1939): "L'état de guerre d'une part, et les nécessités de l'hébergement des populations françaises d'autre part, rendent plus que jamais désirable le retour en Espagne du plus grand nombre possible de réfugiés espagnols et surtout des éléments non susceptibles d'apporter à l'économie française le concours d'un travail utile».

2. A. Egido León, "Republicanos espańoles en la Francia de Vichy. Mano de obra para el invasor ", Ayer, n 46, 2002, p. 189-208.

3. Convention $n^{\circ} 29$ de l'OIT concernant le travail forcé ou obligatoire adoptée à Genève le 28 juin 1930.

4. Article 2 de la Convention de l'OIT du 28 juin 1930. 
en faisant valoir son accord de principe, ne voulait pas remettre en cause la pratique du travail forcé dans son empire colonial. Il fallut attendre le 24 juin 1937 pour que le Front Populaire se décide enfin à ratifier cette décision ${ }^{5}$.

On a souvent tendance à considérer le travail forcé comme l'apanage de l'Allemagne du Troisième Reich ou de la Russie de Staline, en oubliant un peu trop vite qu'il exista dans de nombreux pays pendant la période de l'entredeux-guerres comme un moyen politique pour enrayer la crise économique. Toutes les grandes puissances coloniales - l'Allemagne en Namibie, la GrandeBretagne en Afrique du Sud - y eurent massivement recours. Dans les années 1930, treize états, dont la Bulgarie, la Hongrie, la Roumanie et le Japon, instaurèrent des formes variées de travail obligatoire au nom du « redressement national ", de l' " utilité nationale » ou du "sentiment national » ${ }^{6}$. La France de la Troisième République n'échappe pas à la règle. En Afrique occidentale française $(\mathrm{AOF})$, elle mit en place un système d'exploitation humaine et économique d'une redoutable efficacité.

Grâce aux travaux de l'historien Babacar Fall, cette page peu glorieuse de l'histoire coloniale nous est aujourd'hui relativement bien connue ${ }^{7}$. Entre 1900 et 1946, les indigènes des colonies furent victimes d'une terrible exploitation économique que l'on s'efforçait de justifier par la vertu éducative du travail, antidote de l'oisiveté perverse, et le succès de l'entreprise coloniale, censée apporter la civilisation et le progrès à des êtres primitifs et barbares. Selon les lieux et les époques, ce système prit cinq formes distinctes: la réquisition de la main-d'œuvre indigène, la prestation, la deuxième portion du contingent, la main-d'œuvre pénale et "l'obligation de cultiver " 8 .

La loi du 18 juillet 1938 sur "l'organisation générale de la nation pour le temps de la guerre » instaura le principe de la réquisition pour les Français et les ressortissants français du sexe masculin âgés de plus de 18 ans qui devaient obligatoirement se faire recenser à la mairie de leur lieu de résidence, en indiquant clairement leur nom, leur adresse et leur profession? ${ }^{9}$ Prévue dès le temps de paix, cette mesure d'exception serait mise à exécution en cas de tension extérieure ou d'agression manifeste mettant le pays dans l'obligation de se défendre:

Article 14. À la mobilisation ou dans les cas prévus à l'article 1er de la présente loi, les Français et ressortissants français du sexe masculin, âgés de plus de dix-huit ans, même soumis aux obligations militaires définies par la loi de recrutement et par l'article 11 de la présente loi, sous réserve qu’ils ne soient pas utilisés par les ministres

5. B. Fall, «Le travail forcé en Afrique occidentale française (1940-1946) », Civilisations, 41 (1993), p. 4 [article en ligne].

6. P. Gaida, Camps de travail sous Vichy. Les "Groupes de Travailleurs étrangers " (GTE) en France et en Afrique du Nord 1940-1944, Paris, 2007. Thèse inédite soutenue sous la direction de Denis Peschanski (Paris I), p. 5-7.

7. B. Fall, Le travail forcé en Afrique Occidentale Française 1900-1946, Paris, Karthala, 1993.

8. B. Fall, « Le travail forcé en Afrique occidentale française (1940-1946) ", op. cit., p. 1-5.

9. Journal Officiel du 13 juillet 1938. Loi sur l'organisation générale de la nation pour le temps de la guerre. 
intéressés, peuvent être requis dans les conditions fixées par la loi du 3 juillet 1877 , modifiée par la loi du 21 janvier 1935 (sous réserve des dispositions prévues à l'article 27 de la présente loi), par la loi du 31 mars 1928 et par la présente loi. L'appel sous les drapeaux fait cesser la réquisition. La réquisition est temporaire ou permanente. Les requis sont utilisés suivant leur profession et leurs facultés, ou, s'il y a lieu, suivant les aptitudes, en commençant par les plus jeunes et en tenant compte de la situation de famille, soit isolément, soit dans les administrations et services publics, soit dans les établissements et services fonctionnant dans l'intérêt de la nation. Les requis non soumis aux obligations militaires définies par la loi de recrutement ne peuvent, dans aucun cas, être affectés aux corps spéciaux. L’article 40 de la loi du 13 juillet 1927 est abrogé dans celles de ses dispositions qui sont contraires aux présentes. Peut être également soumis à réquisition, chaque individu conservant sa fonction ou son emploi, l'ensemble du personnel faisant partie d'un service ou d'une entreprise considérée comme indispensable pour assurer les besoins du pays ${ }^{10}$.

La réquisition s'appliquait non seulement aux personnes, mais aussi aux animaux, aux biens et aux divers services de transports et de transmissions. À tout moment, l'État pouvait prendre la décision de nationaliser la fabrication des matériels de guerre, suspendre l'exportation, l'importation ou la circulation de certains produits, interdire la vente de certaines ressources, les taxer ou les rationner. Tant qu'ils n'étaient pas appelés sous les drapeaux, tous les citoyens $-\mathrm{y}$ compris les personnes retraitées depuis moins de cinq ans et les chômeurs volontaires - pouvaient être requis à titre individuel ou collectif dans les administrations et les services de l'État, ou dans les entreprises privées fonctionnant dans l'intérêt de la nation. Sous l'autorité du ministre du Travail chargé de la répartition de la main-d'œuvre, les préfets assureraient la répartition des ressources en personnel entre le secteur public et le secteur privé, en donnant la priorité aux établissements travaillant pour les armées et participant à la défense de la nation.

En vertu de cette loi dictée par les nécessités de l'économie et mue par une idéologie patriotique, tous les Français étaient astreints au régime des prestations et obligés de participer à l'effort de guerre exigé par la nation. Les réfugiés bénéficiaires du droit d'asile, les apatrides et les étrangers résidents en étaient exclus, mais ils avaient la possibilité toutefois de "s'engager » à titre volontaire, comme le stipule expressément l'article 19:

Article 19. À la mobilisation ou dans les cas prévus à l'article $1^{\text {er }}$ de la présente loi, des décrets fixent les conditions dans lesquelles les sujets étrangers peuvent être admis, sur leur demande écrite, à apporter leur collaboration aux administrations, établissements et services prévus à l'alinéa 3 de l'article 14 .

La préparation des mesures devant faire l'objet de ces décrets est prévue dans des instructions arrêtées dès le temps de paix, à la diligence des ministres intéressés.

En ce qui concerne l'emploi, comme main-d'oeuvre, des ressortissants alliés ou neutres stationnés en France, des instructions déterminent, dès le temps de paix également, les départements ministériels compétents pour régler la situation de ces étrangers: 
$1^{\circ}$ Vis-à-vis des autorités de leur propre pays ;

$2^{\circ}$ Vis-à-vis des lois et autorités françaises et pour fixer les règles de leur utilisation ${ }^{11}$.

Le 20 mars 1939, un décret appliquait les dispositions de la loi du 18 juillet 1938 aux réfugiés espagnols et, le 12 avril 1939, le gouvernement Daladier publia un "décret relatif à l'extension aux étrangers bénéficiaires du droit d'asile des obligations imposées aux Français par les lois de recrutement et la loi sur l'organisation de la Nation en temps de guerre $»^{12}$. Ce texte, voté par les parlementaires et approuvé par le Tribunal militaire de Cassation de Paris, imposa à tous les étrangers bénéficiaires du droit d'asile et aux apatrides âgés de 20 à 48 ans les mêmes obligations qu'aux Français, soumis au recrutement militaire et au régime de la réquisition ${ }^{13}$. Pour faire face au manque de maind'œuvre pour la défense nationale et pour mettre les étrangers à égalité de devoirs avec les Français, le droit d'asile fut désormais subordonné à une mobilisation économique sous forme de prestations:

Article 2: Les étrangers sans nationalité et les autres étrangers bénéficiaires du droit d'asile, sont soumis à toutes les obligations imposées aux Français par la loi du 11 juillet 1938 sur l'organisation de la nation en temps de guerre. Ils peuvent faire l'objet de réquisitions individuelles ou collectives, générales ou locales, fondées sur la nationalité, sur l'âge ou sur la profession.

Article 3: Les étrangers sans nationalité et les autres étrangers bénéficiaires du droit d'asile, du sexe masculin, sont assujettis, de vingt à quarante-huit ans, dans les conditions fixées par les lois de recrutement, à fournir, dès le temps de paix aux autorités françaises, pour une durée égale à la durée du service imposé aux Français, des prestations dont le caractère et le mode d'exécution sont déterminées par décret. La durée des services accomplis dans un corps de l'armée française, soit en vertu de la loi du 31 mars 1928, soit en vertu d'un engagement contracté, par l'application de la loi du 9 mars 1831 ou de l'article 64 de la loi du 31 mars 1928, compte dans la durée des prestations imposées par l'alinéa qui précède ${ }^{14}$.

Les réfugiés devenaient ainsi des prestataires au même titre que les requis français. Ceux qui refusaient de se soumettre à ce régime d'exception, perdaient ipso facto le bénéfice du droit d'asile et l'autorisation de résider en France. En utilisant les réfugiés comme des travailleurs forcés, le décret-loi du 12 avril 1939 légalisait la création des CTE dont le cadre statutaire fut fixé par un décret du 27 mai 1939.

\section{L'ORGANISATION DES COMPAGNIES DE TRAVAILLEURS ÉTRANGERS}

Les réfugiés étaient recrutés par voie d'appel ou par voie d'engagement volontaire. Les opérations de recrutement étaient centralisées à Paris au bureau

11. Journal Officiel du 13 juillet 1938, p. 2.

12. Journal Officiel du 16 avril 1939, p. 4910.

13. Service Historique de l'Armée de Terre (SHAT), 31N53. Note $n^{\circ} 1790$ du général Gamelin du 20 avril 1939.

14. Journal Officiel du 16 avril 1939, p. 4910. 
de recrutement des étrangers prestataires situé à l'École militaire ${ }^{15}$. Par les soins de ce bureau, les intéressés étaient convoqués, dotés d'un livret individuel et mis en route sur les dépôts de formation des unités de travailleurs étrangers.

Les prestataires pouvaient être incorporés en qualité de travailleurs encadrés, de travailleurs isolés rattachés à certaines compagnies, ou placés, dans certains cas, en position d'affection individuelle. Ce dernier régime équivalait presque à l'exemption des prestations, et ne devait être réservé, en principe, qu'à des " personnalités intellectuelles dont le loyalisme à l'égard de la France ne peut être mis en doute et dont les capacités peuvent être utilisées dans l'intérêt national à des fins autres que l'exécution des tâches manuelles " ${ }^{16}$. Les demandes étaient instruites et adressées, par l'intermédiaire des divers départements ministériels, au ministre de la Guerre en vue de leur examen par la Commission des affectations individuelles qui statuait sur les demandes, se prononçait sur les radiations et donnait son avis, à titre consultatif, sur toutes les questions relatives au placement et à l'utilisation des prestataires. Les " affectés " individuels figuraient sur un fichier spécial et faisaient l'objet d'un recensement à part. Dégagés du régime des prestations, ces derniers ne pouvaient se prévaloir de leur statut pour bénéficier des avantages prévus en faveur des travailleurs étrangers en matière de solde, de transport en chemins de fer, de législation sur les loyers, etc.

Les prestataires se trouvaient sous la tutelle du ministre de la Défense et de la Guerre, en vue de l'accomplissement des prestations dans les conditions fixées par les textes en vigueur. À ce titre, ils étaient régulièrement incorporés en qualité de travailleurs étrangers et soumis, en ce qui concerne le régime disciplinaire, aux dispositions du code de justice militaire pour l'armée de Terre en vertu du décret du 9 avril $1940^{17}$. Ils recevaient une instruction appropriée à leur état visant à leur inculquer les notions indispensables sur la discipline, l'organisation du service et la situation des travailleurs étrangers ; à leur permettre la bonne exécution des rassemblements et déplacements nécessaires aux services qui leur étaient confiés; et à leur apprendre à se protéger contre les gaz et les bombardements aériens. Les travailleurs étrangers devenus inaptes au service des prestations passaient devant une Commission de réforme dans les mêmes conditions que les militaires français.

Les CTE étaient constituées en sections de nombre variable suivant les circonstances. En général, il y en avait cinq de cinquante prestataires chacune, soit au total 250 hommes, parmi lesquels figuraient les employés permanents de l'unité. L'encadrement comprenait un officier du grade de capitaine, lieutenant ou sous-lieutenant, commandant la compagnie ; cinq sous-officiers, chefs des

15. ADH, 15W1. Instruction sur l'organisation et l'utilisation des travailleurs étrangers (prestataires) en temps de guerre, p. 1. L'École militaire se trouvait à l'adresse suivante: 14 Place Joffre.

16. $\mathrm{ADH}, 15 \mathrm{~W} 1$. Instruction sur l'organisation et l'utilisation des travailleurs étrangers (prestataires) en temps de guerre, p. 30.

17. ADH, 15W1. Instruction sur l'organisation et l'utilisation des travailleurs étrangers (prestataires) en temps de guerre, p. 3. 
sections de travailleurs ; cinq caporaux ; un sous-officier comptable ; et un caporal comptable. Ces cadres servaient au titre français ou au titre étranger, et continuaient à appartenir à leur arme d'origine dont ils étaient détachés. Les sous-officiers et caporaux devaient, en temps de guerre, appartenir aux classes de la deuxième réserve. Ils étaient équipés de la tenue de campagne et armés de revolvers. Ils maintenaient l'ordre et la discipline, assuraient la garde des travailleurs sur les chantiers et au cantonnement, et s'efforçaient d'obtenir un rendement de travail maximal lorsque la surveillance des travaux leur incombait ${ }^{18}$. En cas d'indiscipline, de mauvaise volonté au travail ou de propagande antinationale, les travailleurs étaient conduits dans des compagnies spéciales, au régime de travail extrêmement sévère, ou renvoyés dans des camps d'internement (camp répressif du Vernet).

Deux secrétaires, trois cuisiniers, deux infirmiers, un cycliste, un coiffeur, un tailleur, un bottier, un ordonnance ${ }^{19}$, un interprète, un ou deux chauffeurs, constituaient les employés permanents de l'unité. Ce personnel était prélevé directement sur les étrangers les moins aptes aux tâches manuelles ou, à défaut, sur du personnel français fourni par le dépôt. Lorsque le nombre des sections était inférieur à cinq, les cadres et les employés diminuaient en conséquence. Par contre, les unités dont les sections n'étaient pas groupées demandaient une vigilance accrue, et celles affectées à des travaux particuliers pouvaient nécessiter la présence d'un deuxième officier chargé de seconder le commandant de compagnie. Il appartenait au général de Région de demander au ministre de la Guerre la désignation de cet officier supplémentaire.

La dotation d'une compagnie comportait une bicyclette, une sacoche de comptable, une caisse de comptabilité, une caisse d'ouvrier bottier, une caisse d'ouvrier tailleur, un jeu d'outils de coiffeur, vingt sacs à distribution, un moulin à café, un jeu de petit matériel de cuisine, un jeu de matériel de distribution, dix marmites de peloton complètes et vingt seaux en toile. Les compagnies qui se déplaçaient souvent, comme celles mises à la disposition des armées, recevaient en outre une camionnette de 1500 kilos à vivres et à bagages, une camionnette de 1500 kilos de ravitaillement, ainsi qu'une cuisine roulante. Dans les compagnies plus stables affectées à des usines d'armement, par exemple, la cuisine était remplacée par des fourneaux auxiliaires. Dans tous les cas, les cadres, officiers et sous-officiers recevaient le matériel de campement nécessaire pour vivre en popote.

Pour leur alimentation, les compagnies constituaient des ordinaires. Lorsque l'unité était réunie, les sous-officiers et caporaux du cadre français pouvaient choisir de vivre à l'ordinaire ou de former un mess particulier. À titre exceptionnel, et principalement dans la section ou compagnie de rattachement d'isolés, le commandant d'unité pouvait autoriser les travailleurs étrangers à

18. ADH, 15W1. Instruction sur l'organisation et l'utilisation des travailleurs étrangers (prestataires) en temps de guerre, p. 10.

19. L'ordonnance est un domestique militaire, un soldat attaché à un officier ou un officier attaché à un commandant en chef. 
ne pas vivre à l'ordinaire, en raison de l'éloignement de leur lieu de travail ou de la nature de leurs occupations. Les travailleurs isolés percevaient alors les prestations d'alimentation en même temps que la solde.

Les travailleurs étrangers avaient droit à une solde journalière de 50 centimes, à une prime de rendement ${ }^{20}$, à une ration mensuelle de tabac fixée à 160 cigarettes ou 200 grammes de scaferlati, à deux timbres spéciaux par mois surchargés " $\mathrm{F}$ » pour l'affranchissement de lettres simples et de cartes postales à destination du territoire français, ainsi qu'aux allocations militaires pour leurs familles ${ }^{21}$. À l'instar de n'importe quel soldat accomplissant la durée légale du service, ils bénéficiaient de permissions qui, en raison des conditions particulières de leur service, étaient établies de la façon suivante :

-permissions exceptionnelles de trois jours au plus, prévues pour les cas graves (maladies, décès d'un proche, etc.) ;

-permissions de détente accordées pour une destination unique de la France continentale, à raison de quinze jours d'affilée ou de deux fois une semaine par an ;

-permissions de convalescence d'une durée de huit jours maximum.

Les travailleurs étrangers occupés dans l'industrie ou l'agriculture pouvaient, lorsqu'ils donnaient entière satisfaction et notamment quand leurs familles résidaient à proximité, être autorisés à bénéficier de permissions de la journée ou de vingt-quatre heures les jours de repos, lorsque les nécessités du service ou de la surveillance le permettaient. Toutes ces permissions donnaient droit au transport gratuit en troisième classe, aux délais de route à l'aller et au retour et, pendant toute la durée du congé, à la solde et aux indemnités militaires diverses dont le montant était versé avant le départ. L'obtention d'une permission était toutefois subordonnée à la présentation d'un certificat d'hébergement délivré par le commissaire de police ou, à défaut, le maire du lieu de résidence de la famille de l'intéressé.

Le Service de santé militaire assurait les visites d'incorporation, les visites médicales, les soins aux malades et aux blessés et, éventuellement, l'hospitalisation dans les formations sanitaires. Les infirmiers des compagnies ne pouvant assurer que le service courant, il appartenait aux généraux commandant les Régions pour les formations stationnées à l'intérieur, et aux généraux commandant les grandes unités pour les formations mises à leur disposition dans la zone des armées, d'organiser le service médical des unités de travailleurs avec les moyens du bord.

20. Cette prime de rendement s'avérait, dans bien des cas, être davantage une fiction qu'une réalité.

21. ADH, 15W1. Instruction sur l'organisation et l'utilisation des travailleurs étrangers (prestataires) en temps de guerre, p. 13: "Les allocations militaires sont attribuées aux familles des Travailleurs étrangers reconnues nécessiteuses, conformément à la réglementation en vigueur et notamment aux dispositions des Décrets du 1er septembre 1939 et du 6 novembre 1939. Seules les familles résidant en France, Algérie, Colonies, pays de protectorat ou territoires sous mandat français, peuvent bénéficier de ces dispositions et les allocations cessent d'être versées lorsque les familles quittent ces territoires ». 
L'utilisation des prestataires comportait l'emploi en unités constituées, encadrées et surveillées au sein des CTE. Pour obtenir une rentabilité maximale de la main-d'œuvre, il fallait que ces formations soient adaptées aux nécessités économiques locales ainsi qu'aux divers besoins nationaux. C'est la raison pour laquelle les compagnies étaient constituées sur les instructions du ministre de la Défense et de la Guerre et à la diligence des commandants de Région, en tenant compte des grandes catégories professionnelles d'intérêt national : mine, métallurgie, industrie électrique, aviation, bâtiment et agriculture. Une compagnie pouvait être utilisée en sections de même catégorie professionnelle dans des établissements voisins, ou en sections de professions différentes, notamment dans les agglomérations abritant plusieurs branches industrielles. Pour répondre au mieux aux nécessités de l'économie, les sections pouvaient comprendre, outre des spécialistes, une proportion de manœuvres d'industrie non qualifiés, correspondant aux besoins normaux des établissements. Dans les grands centres, on pouvait prévoir la constitution de compagnies de rattachement d'isolés travaillant par petits groupes ou à titre individuel. Une fois épuisées les ressources professionnelles, on procédait à la formation d'unités sans spécialistes, destinées à des travaux d'ordre courant et de manœuvres. En général, on groupait les travailleurs suivant leurs aptitudes physiques en vue de leur spécialisation à des travaux plus ou moins durs: compagnies de manœuvres de force, de terrassiers, de manutentionnaires, de maçons, etc ${ }^{22}$.

Les unités constituées étaient mises par le ministre de la Défense et de la Guerre à la disposition des généraux commandant les Régions, de certains départements ministériels et, éventuellement, du général commandant en chef, pour l'exécution de travaux d'intérêt général, en rapport avec leurs capacités professionnelles. Elles pouvaient être utilisées soit par des administrations ou services de l'État, soit par des employeurs, industries, établissements ou entreprises, personnes morales ou civiles, travaillant pour la défense nationale, à charge de remboursement à l'État des salaires gagnés. Dans ce dernier cas, les unités dépendaient, pour le contrôle de leur utilisation technique, d'un établissement d'État dit de rattachement.

Le ministre de la Défense nationale et de la Guerre pouvait, à titre exceptionnel, mettre à la disposition du ministère de l'Agriculture des travailleurs étrangers sous la forme :

-1) de compagnies de travailleurs étrangers plus ou moins spécialisées en travaux courants et saisonniers de l'agriculture, travaillant en formations complètes ( 250 hommes), en sections ( 50 hommes) ou en équipes (groupes de moins de 50 hommes). Les travailleurs touchaient la solde journalière ainsi qu'une prime de rendement individuelle dont le montant était fixé suivant la nature des travaux, les conditions de travail et le rendement obtenu, par accord entre l'employeur et le commandant d'unité. Ceci dit, le taux horaire de cette prime ne devait pas excéder 50 centimes. En vue

22. ADH, 15W1. Instruction sur l'organisation et l'utilisation des travailleurs étrangers (prestataires) en temps de guerre, p. 19. 
du remboursement des salaires par l'employeur, le taux de la journée de travail était arrêté par le préfet, en accord avec le général commandant la Région militaire et l'inspecteur départemental du Travail, sur la base de $75 \%$ du salaire régional, "pour tenir compte de la qualité professionnelle d'une main-d'œuvre non spécialisée et en y incorporant les frais annexes de charges sociales normalement à la charge de l'employeur $"^{23}$. L'employeur remboursait intégralement le salaire à l'État, après déduction des sommes versées au commandant d'unité à titre de primes de rendement ;

-2) d'isolés, de cultivateurs, d'ouvriers agricoles ou de travailleurs de l'artisanat rural ${ }^{24}$ maintenus, en principe, dans leur exploitation ou, suivant les besoins locaux, travaillant individuellement ou par petits groupes. Chaque département possédait une ou deux compagnies de rattachement d'isolés. Le commandant de l'unité n'avait plus que des fonctions de contrôle et de surveillance sur ces hommes, ce qui fait que les employés permanents pouvaient être réduits à deux secrétaires, un cuisinier, un cycliste et un ordonnance. De même, la dotation en matériel ne comprenait plus qu'une bicyclette, une caisse de comptabilité et une sacoche de comptable. Les travailleurs ainsi détachés dans l'ensemble du département ne recevaient plus de solde, ni d'indemnité, ni de fournitures d'aucune sorte et pourvoyaient eux-mêmes à leur subsistance et à leur entretien. Ils ne pouvaient plus prétendre aux avantages accordés aux militaires, mais continuaient à bénéficier, en revanche, de la législation sociale. Ils devaient se consacrer entièrement aux travaux agricoles de leur profession, mais étaient astreints, en outre, à pratiquer l'entraide sous le contrôle du Comité départemental de la production agricole et du commandant d'unité. Cette entraide obligatoire consistait, en principe, à fournir deux jours de prestations par semaine à d'autres cultivateurs de la commune et en priorité aux exploitations des mobilisés.

Le contrat individuel ou collectif passé entre l'employeur et le commandant de la compagnie stipulait les conditions de travail des prestataires, les catégories professionnelles, la base des indemnités à verser à l'État et les modalités de remboursement. Les travailleurs étrangers ne pouvaient être mis à la disposition de départements ministériels qu'après reconnaissance par l'autorité militaire de la possibilité d'assurer leur subsistance et leur cantonnement. Celui-ci devait présenter les conditions de confort et de salubrité nécessaires et la possibilité d'installer les bureaux d'unités, les cuisines et, éventuellement, des douches si l'établissement employeur n'en mettait pas à la disposition de son personnel. $\mathrm{Si}$ les seuls cantonnements acceptables à proximité de l'établissement

23. $\mathrm{ADH}, 15 \mathrm{~W} 1$. Instruction sur l'organisation et l'utilisation des travailleurs étrangers (prestataires) en temps de guerre, p. 20.

24. ADH, 15W1. Instruction sur l'organisation et l'utilisation des travailleurs étrangers (prestataires) en temps de guerre, p. 21: "La définition des artisans ruraux est fixée par la nomenclature adoptée pour l'application de la loi sur les allocations familiales en agriculture (maréchaux-ferrants, forgerons, réparateurs de machines agricoles, bourreliers, sabotiers, tonneliers, charrons". 
appartenaient à l'employeur, ce dernier était tenu de les mettre à disposition des unités. S'il s'agissait d'un employeur privé, assujetti au remboursement des salaires à l'État, l'utilisation des locaux par la troupe lui donnait droit à toucher des indemnités. Si les emplacements respectifs du cantonnement et de l'établissement exigeaient des transports exceptionnels, l'imputation des frais était fixée dans le contrat, en tenant compte des possibilités locales et des moyens dont disposait l'employeur.

Le salaire de base dans l'industrie et dans les entreprises était établi par accord entre l'employeur et le commandant de compagnie, assisté par l'inspecteur du Travail, et soumis à l'approbation, suivant les cas, du directeur de l'établissement d'État de rattachement ou du représentant local du ministère utilisateur : ingénieur en chef des Mines, des Ponts et Chaussées, etc. Sur la base du salaire moyen, on calculait le revenu brut sans retenue fiscale ou sociale, par journée de travail normale. En cas de désaccord entre l'employeur et le commandant de compagnie, celui-ci en référait au général commandant la Région militaire. L'employeur avait l'obligation de rembourser à l'État le montant total des salaires gagnés par les travailleurs qu'il occupait, augmenté des charges diverses qui lui reviendraient en temps normal. Chaque quinze jours, il remettait au commandant de la compagnie, le montant de la somme qui allait au Trésor public dans la rubrique "Recettes accidentelles à différents titres ${ }^{25}$.

Une prime individuelle de bon rendement était allouée aux travailleurs étrangers employés en usine, sur des chantiers spéciaux ou dans les mines, à l'exclusion des travaux accomplis normalement par les bataillons de pionniers, les sapeurs du Génie ou unités similaires de l'Armée. Elle ne pouvait faire l'objet d'aucune retenue de la part de l'unité. Le taux de cette prime était variable, suivant le rendement de chacun, la catégorie professionnelle, la nature du travail et l'industrie. Le calcul se faisait par accord entre l'employeur et le commandant de compagnie après avis du directeur d'État de rattachement ou du représentant local du ministère utilisateur. Il prenait en considération les maxima suivants :

-dans l'industrie : manœuvres en usine légèrement spécialisés (75 centimes par heure), professionnels d'aviation fortement spécialisés (1,50 franc par heure).

-dans les mines : mineurs à l'abattage les mieux payés (15 francs par journée).

Autant dire que les travailleurs étrangers faisaient l'objet d'une exploitation systématique. Dans le meilleur des cas, solde journalière et prime de rendement comprises, ils arrivaient péniblement à toucher 300 francs, somme dérisoire eu égard au salaire d'un ouvrier agricole français qui tournait autour des $1000 \mathrm{ou}$ 1100 francs par mois.

25. ADH, 15W1. Instruction sur l'organisation et l'utilisation des travailleurs étrangers (prestataires) en temps de guerre, p. 27. 
LE FONCTIONNEMENT DES COMPAGNIES DE TRAVAILLEURS ÉTRANGERS

Dès le printemps, le gouvernement français songea à mettre au travail les réfugiés espagnols hébergés sur son territoire, afin de faire face à la pénurie de main-d'œuvre provoquée par le départ des mobilisés, de réduire la population des camps d'internement, de favoriser les regroupements familiaux, et d'alléger les charges d'assistance de l'État qui se montaient à huit cents millions de francs pour la seule année $1939^{26}$. Dans le même temps, il encouragea les " réémigrations " à destination du Mexique et de l'Amérique Latine, et le rapatriement « des personnes n'ayant rien à redouter d'un retour en Espagne et qui n'ont cherché refuge en France que sous l'effet de la panique qui a déferlé en Catalogne dans les derniers jours de janvier ${ }^{27}$.

À partir de la déclaration de guerre, la seconde attitude finit par l'emporter et l'on mit tout en place pour retenir cette main-d'œuvre étrangère jugée indispensable pour le redressement de l'économie nationale :

Depuis la Retirada, les pouvoirs publics ont oscillé entre deux politiques à l'égard des réfugiés espagnols: leur renvoi à l'extérieur du territoire français afin d'alléger les dépenses occasionnées par leur arrivée et leur utilisation comme main-d'œuvre ou comme force combattante afin de tirer profit de leur présence. À partir de la déclaration de guerre, la seconde attitude - esquissée dans la circulaire du 19 septembre - prévaut indubitablement, mais le souci de se débarrasser au plus tôt de ceux qui ne peuvent être utiles à l'économie française demeure présent. Cependant, au cours du premier semestre de 1940, la main-d'œuvre espagnole apparaît si indispensable que, par deux fois, la date d'application des instructions de février 1940 est repoussée: fixée d'abord au 15 mars, la date est reportée au $1^{\text {er }}$ mai puis au $1^{\text {er }}$ juin. Il est fait mention d'une « main-d'œuvre qui s'avère de jour en jour plus indispensable ». Au mois de mai, il est même question que les hommes, les femmes et les enfants âgés de plus de quatorze ans, aptes à un travail manuel, devront être autorisés à demeurer en France, à moins qu'ils ne soient dangereux pour l'ordre public ${ }^{28}$.

Comme l'expliquait le ministre de l'Intérieur Albert Sarraut aux préfets au mois de mai 1939, il fallait que les réfugiés cessent d'être assistés et passent sous le régime des étrangers de droit commun, et " transformer cette masse inorganisée et passive que constituent les réfugiés en éléments utiles à la collectivité nationale ${ }^{29}$. Si l'hospitalité française restait " ouverte à qui apporte à la nation le concours de son travail ", l'intérêt supérieur du pays commandait "qu'elle soit désormais fermée aux oisifs et aux inutiles »"

C'est ainsi que 40000 réfugiés furent placés à titre individuel par le ministère du Travail dans l'agriculture, l'industrie, les mines, les houillères, les usines

26. P. Gaida, Camps de travail sous Vichy. Les " Groupes de Travailleurs étrangers " (GTE) en France et en Afrique du Nord 1940-1944, op. cit., p. 19.

27. ADH, 2W602. Circulaire du ministère de l'Intérieur aux préfets (5/05/1939).

28. G. Dreyfus-Armand, L'exil des républicains espagnols en France. De la Guerre civile à la mort de Franco, Paris, Albin Michel, 1999, p. 109.

29. ADH, 2W602. Circulaire du ministère de l'Intérieur aux préfets (5/05/1939).

30. ADH, 2W602. Circulaire n 515 du ministre de l'Intérieur (13/01/1940). 
aéronautiques, les forges, les poudreries, les entreprises d'armement, etc., et que 55000 furent incorporés à titre collectif dans les CTE. En juillet 1940, dans le département de la Haute-Garonne, trois entreprises employaient une abondante main-d'œuvre espagnole: 1500 à la Poudrerie nationale de Toulouse, 1300 à la Poudrerie du Fauga et 1600 à la Société nationale des constructions aéronautiques du $\mathrm{Midi}^{31}$. De février à août 1939, le bassin minier de Decazeville recruta plus de 300 réfugiés qui vinrent s'ajouter aux 268 mineurs espagnols sédentaires qui avaient émigré dans l'Aveyron avant la Guerre civile $^{32}$. Les Compagnies de Saint-Etienne et les mines de la GrandCombe dans le Gard puisèrent à leur tour dans les camps une importante main-d'œuvre de mineurs et de travailleurs agricoles ${ }^{33}$. À la fin du mois de janvier 1940, 2906 miliciens du camp de Septfonds avaient été placés dans l'agriculture et 1087 dans l'industrie ${ }^{34}$.

Cependant, aux yeux des autorités militaires, l'utilisation collective de la main-d'œuvre étrangère s'avérait de loin préférable au placement individuel qui pouvait faire courir un danger pour l'ordre public et la sûreté nationale. C'est ce qui ressort très nettement du rapport en date du 21 octobre 1939 du capitaine Barrié, interprète du camp de Septfonds:

Les miliciens sont tous de plus en plus préoccupés de leur sortie du camp et cherchent leur voie dans les nombreuses catégories de travailleurs où on les classe. Ils s'offrent indifféremment pour des métiers qui ne sont certainement pas toujours les leurs, très souvent pour plusieurs à la fois. Ils veulent tous rester à tout prix en France pour s'y occuper et s'y incorporer, et ils donnent trop souvent l'impression de considérer le camp comme un bureau de placement. Ainsi, la compagnie de travailleurs encadrée semble-t-elle être la meilleure forme d'utiliser la main-d'œuvre comme il a été exposé dans plusieurs rapports précédents [...] notamment pour permettre l'utilisation d'un grand nombre de réfugiés dont les aptitudes sont mal définies, diverses et de valeur médiocre. Les sorties individuelles font courir des risques à l'ordre et à l'état d'esprit. Les militants communistes et anarchistes peuvent, une fois isolés et inconnus, recommencer à se livrer à une propagande contre la France et son gouvernement et servir d'agents bons à tout faire contre la défense nationale ${ }^{35}$.

Il va sans dire que les conditions de vie du réfugié recruté individuellement et celles du réfugié embauché pour divers travaux collectifs n’avaient absolument rien à voir. Le premier jouissait d'une relative liberté, vivait parfois avec sa famille lorsque ses revenus le lui permettaient, tandis que le second était condamné à vivre en célibataire et soumis à une discipline militaire et à des conditions de travail extrêmement rudes. Très souvent, les cantonnements installés dans des bâtiments de ferme, des friches industrielles, des étables, des locaux militaires, etc., n'offraient pas les conditions sanitaires et hygiéniques minimales requises

31. G. Dreyfus-Armand, L'exil des républicains espagnols en France, op. cit., p. 112.

32. B. Bennassar, La guerre d'Espagne et ses lendemains, Paris, Perrin, 2004, p. 435.

33. B. Bennassar, "L'apport des réfugiés à l'économie (1939-1941) ", in Républicains espagnols en Midi-Pyrénées. Exil, histoire, mémoire, Toulouse, PUM, 2005, p. 155-162.

34. B. Bennassar, "L'apport des réfugiés à l'économie (1939-1941)", op. cit., p. 160.

35. B. Bennassar, La guerre d'Espagne et ses lendemains, op. cit., p. 432. 
pour une vie en collectivité. C'est avec une amertume non dénuée d'humour que Martin Armingol évoque, dans ses mémoires, son séjour dans une CTE dans le Cher près de la ville d'Aubigny. Écoutons ses propos:

Notre terminus fut une petite gare du Cher. On nous conduisit dans une grande ferme où nous fûmes installés dans des hangars, étables et autres dépendances. La ville la plus proche s'appelait Aubigny.

La faim nous tenaillait; le propriétaire et son domestique arrivèrent avec un cheval attelé à un tombereau rempli de raves bien propres [...]. Le lendemain, accompagnés par un sergent qui mesurait deux mètres, nous allâmes couper des arbres qui bordaient le chemin de la propriété. Soixante-dix pour cent des hommes qui composaient notre " compagnie de travail " n'étaient pas agriculteurs et ne savaient pas se servir d'une hache ou d'une scie. Ce pauvre sergent nous prit les outils des mains pour nous expliquer comment procéder mais, voyant notre indifférence, exaspéré, il jeta son calot et le piétina de colère, nous menaçant avec un bâton. Il ne savait pas à qui il avait affaire. Pour finir de le démoraliser, toute la compagnie chanta l'Internationale. Il frappa le sol, mais ne toucha personne $e^{36}$.

En dépit des vœux pieux du gouvernement qui encourageait les regroupements familiaux, pour des raisons - ne l'oublions pas - avant tout budgétaires, les travailleurs étrangers continuèrent à vivre, de longs mois durant, séparés de leurs femmes et de leurs enfants. En décembre 1939, Jean Moulin alors préfet d'Eure-et-Loir faisait savoir, à la suite de bien d'autres préfets, qu'il se trouvait dans l'impossibilité de faire venir dans son département les familles des réfugiés espagnols du camp de Bram appelés à suppléer temporairement le manque de main-d'œuvre dans l'agriculture et employés aux battages, car ils ne touchaient pas de salaire mais une indemnité journalière variant entre 5 et 10 francs, autrement dit 300 francs par mois:

Bien que les instructions ministérielles soient favorables à ces regroupements, j'ai l'honneur de vous faire connaître que, malgré mon désir de donner satisfaction à ces réfugiés dont l'appoint en main-d'œuvre m’a été particulièrement précieux, je ne puis envisager d'accorder à ces étrangers l'autorisation qu'ils sollicitent en raison de ce que leurs gains ne constituent pas une rémunération leur permettant d'assurer la subsistance de leur famille. En outre, les travaux pour lesquels ces réfugiés ont été demandés sont sur le point de prendre fin, et il est vraisemblable qu'ils seront obligés de réintégrer leur camp d'origine, après l'échéance ${ }^{37}$.

Les camps du sud-ouest qui fournissaient les CTE se transformèrent ainsi en de véritables offices de placement. Pour mener à bien ce travail d'intégration des Espagnols dans l'économie nationale, on procéda, dès l'été 1939, à un recensement et à un examen d'aptitude professionnelle des internés ${ }^{38}$. À la différence de l'immigration antérieure qui provenait des régions encore très rurales du centre et du centre-est de l'Espagne, la vague migratoire de 1939

36. M. Armingol, Mémoires d'un exilé espagnol insoumis 1931-1992, Paris, L'Harmattan, 1995, p. 154.

37. ADH, 2W602. Lettre de Jean Moulin datée du 13 décembre 1939.

38. ADH, 2W602. Ministre de l'Intérieur aux préfets (1/08/1939). 
venait en grande partie de la Catalogne, région qui, avec le Pays Basque, était l'une des plus riches et des plus industrialisées de la péninsule. Selon les estimations du SERE, environ $45 \%$ des réfugiés appartenaient au secteur industriel, $30 \%$ au secteur primaire, $12 \%$ au secteur tertiaire et $13 \%$ à des secteurs indéfinis ${ }^{39}$. Comme on peut aisément l'imaginer, les ouvriers spécialisés dans le travail des métaux et dans la fabrication de thermomètres, indicateurs de vitesse, contrôleurs de vol, etc., furent particulièrement convoités par l'industrie de guerre et les usines d'armement.

Certaines compagnies restaient sur place pour veiller à l'entretien et au bon fonctionnement des camps, mais la plupart d'entre elles étaient dispersées aux quatre coins de l'Hexagone et en Afrique du Nord, au Maroc, en Tunisie et en Algérie. À titre d'exemple, au début de mai 1940, en dehors des compagnies affectées aux armées, "sept compagnies se trouvaient en Normandie, six en Bretagne, vingt dans le Centre, une dans le Doubs, quinze en Champagne et Bourgogne, vingt-neuf en Poitou-Charentes, sept dans les Pays-de-Loire, trois en Auvergne, six dans l'Ain et l'Isère, cinq dans le Languedoc, les Bouchesdu-Rhône et la Corse, deux dans le Roussillon, quatorze dans la seule HauteGaronne, six dans le Lot, le Lot-et-Garonne et l'Ariège, seize en Aquitaine et une dans l'Oise $»^{40}$. Elles étaient mises à la disposition des différents départements ministériels, et affectées indistinctement aux armées, à l'agriculture, aux entreprises privées et aux services de l'État - Ponts et Chaussées, Eaux et Forêt, Génie, SNCF... - afin de participer à l'organisation défensive des frontières et à l'aménagement de camps militaires.

Dès le mois d'avril 1939, 2500 prestataires espagnols furent employés à la réfection du camp militaire de Suippes dans la Marne41. En mai 1939, le maire de Dax sollicitait l'aide de bûcherons pour des travaux de carbonisation indispensables au bon fonctionnement des gazogènes42. En septembre 1939, deux compagnies formées à partir du camp de Septfonds - CTE 31 et CTE 32 - exécutaient des travaux de fortification sur la ligne Maginot, et construisaient des pièges antichars dans le nord-est de la France ${ }^{43}$. À Toulouse, dix compagnies furent mises à la disposition des industries de guerre ${ }^{44}$. Dans le département du Lot, six compagnies étaient employées aux travaux agricoles, à des aménagements de routes et à la construction de barrages ${ }^{45}$. Au début du mois d'octobre 1939, le maréchal des logis de Sentein (Ariège) accueillit 160 réfugiés venus des camps de Saint-Cyprien, du Barcarès et d'Argelès, pour travailler sur le chantier de haute montagne du lac d'Araing et à l'usine hydroélectrique

39. J. Rubio, La emigración española a Francia, Barcelone, Ariel, 1974, p. 152-154.

40. G. Dreyfus-Armand, L'exil des républicains espagnols en France, op. cit., p. 111.

41. SHAT, 7N429. Rapport n 212 sur le camp d'Espagnols à Suippes (3/06/39).

42. SHAT, 7N327. Lettre du maire de Dax du 15 mai 1940.

43. S. Zorzin, Le camp de Septfonds (Tarn-et-Garonne). Soixante ans d'histoire et de mémoire (1939-1999), Bordeaux, IEP, 2000, p. 38.

44. F. Guilhem, "Les réfugiés espagnols en Haute-Garonne ", in: Républicains espagnols en Midi-Pyrénées. Exil, histoire, mémoire, op. cit., p. 294.

45. G. Dreyfus-Armand, L'exil des républicains espagnols en France, op. cit., p. 311. 
d'Eylie ${ }^{46}$. À Tarascon (Ariège), la CTE 558 employait des ouvriers métallurgistes et des mineurs pour la construction de barrages et l'exploitation de mines. À Saint-Jean-de-Verges (Ariège), la CTE 721 utilisait des manœuvres pour l'agriculture, et à Quérigut (Ariège), la CTE 145 travaillait dans les usines de textile du pays d'Olmes ${ }^{47}$. En décembre 1939, un fort contingent de réfugiés espagnols en provenance du camp de Bram participait à des travaux agricoles (récolte des betteraves) dans le département d'Eure-et-Loir ${ }^{48}$. À l'hiver 1939, plus de quarante compagnies stationnées dans la région des Alpes effectuaient des travaux d'intérêt général, comme le déneigement des cols, la construction de ponts et l'élargissement des routes (Col du Lautaret, par exemple ${ }^{49}$. En avril 1940, l'Union électrique du Sud employait une compagnie de 250 hommes pour les travaux de la chute du Portillon dans le département de la HauteGaronne $^{50}$. À partir du printemps 1940, le président du Conseil décida de remplacer les Français des classes 1914 et 1915 regroupés dans des compagnies de travailleurs militaires, par des prestataires espagnols et de la main-d'œuvre coloniale. Comme le souligne Peter Gaida, il s'agissait, ni plus ni moins, d'une relève des vétérans ${ }^{51}$.

En Afrique du Nord, dans les colonies et les territoires sous protectorat français, les réfugiés espagnols travaillèrent à la construction d'une ligne de chemin de fer dans le sud tunisien, à des réfections de routes dans le sud constantinois, à l'extraction du charbon à Kenadza (Algérie), et à l'aménagement du transsaharien entre Bouarfa, Colomb-Béchar et Kenadza, dans des conditions dignes des forçats ou des bagnards ${ }^{52}$. Comme on peut le constater, le champ d'utilisation des CTE était extrêmement vaste.

Dans un premier temps, les incorporations dans les CTE ne concernaient que les engagés volontaires qui désiraient échapper à l'internement, se débarrasser de l'étiquette d'étrangers "indésirables " qui leur collait désespérément à la peau, et poursuivre la lutte contre le fascisme entreprise contre Franco pendant la Guerre civile. Après la déclaration de guerre, le ministère du Travail interdit tout détachement individuel de prestataires dans l'agriculture, et le volontariat céda la place à un enrôlement obligatoire. Désormais tous les demandeurs du droit d'asile qui remplissaient les conditions fixées dans le décret-loi du 12 avril 1939 durent se soumettre au régime des prestations, s'ils ne voulaient pas se retrouver internés dans un camp ou placés en garde à vue $e^{53}$.

Dans leur grande majorité, les travailleurs étaient des Espagnols, mais l'on trouvait également des ex-membres des Brigades internationales, des

46. B. Bennassar, "L'apport des réfugiés à l'économie (1939-1941)", op. cit., p. 161.

47. J. F. Berdah, "L'Ariège et la question espagnole (1936-1945) ", in: Républicains espagnols en Midi-Pyrénées. Exil, histoire, mémoire, op. cit., p. 281.

48. SHAT, 3P53. Carte de stationnement des CTE (1939-1940).

49. SHAT, 3P53. CTE dans la région des Alpes (hiver 1939-1940).

50. SHAT, 7N327. Lettre de l'Union électrique de France (16/04/1940).

51. P. Gaida, Camps de travail sous Vichy, op. cit., p. 25.

52. G. Dreyfus-Armand, L'exil des républicains espagnols en France, op. cit., p. 116.

53. P. Gaida, Camps de travail sous Vichy, op. cit., p. 22. 
ressortissants des pays ennemis (Allemands, Autrichiens, Dantzigois et Tchécoslovaques), ainsi que des réservistes belges et hollandais. Mention spéciale méritent les réfugiés de droit commun, dont un décret du 4 septembre 1939 prévoyait l'incorporation dans des "sections d'exclus " pour les jeunes condamnés à des courtes peines, et dans des " groupes spéciaux d'étrangers " pour les plus âgés ${ }^{54}$.

Entre le printemps 1939 et l'armistice de juin 1940, le nombre de compagnies ne fit que s'accroître passant respectivement de 53 en avril, à 79 en septembre, à 102 en novembre, à 180 en décembre et à plus de 220 en avril 1940. À cette date, l'état-major de l'Armée faisait savoir que les 104000 ex-miliciens espagnols réfugiés en France avaient tous été astreints au service des prestations : 55000 avaient été organisés en CTE ; 40000 avaient été directement placés par le ministère du Travail dans l'industrie ou l'agriculture ; 6000 s'étaient engagés dans la Légion ou les Régiments de marche de volontaires étrangers; et 3000 inaptes à tout travail avaient été maintenus dans les camps ${ }^{55}$. Les compagnies étaient disséminées dans soixante-sept départements. Les Pyrénées-Orientales arrivaient en tête avec 29 compagnies, suivies par les départements de la Vienne (11), de l'Yonne (10), de la Meuse (9), de la Moselle (9), du Nord (9), de la Gironde (9), du Var (9), des Hautes-Alpes (8), du Tarn-et-Garonne (8), du Loir-et-Cher (7) et du Lot-et-Garonne (7) ${ }^{56}$.

L'histoire des CTE vira au cauchemar et au drame à la fin de la Troisième République. Par un concours de circonstances malchanceux, la trajectoire des prestataires finit par se confondre avec celle des réfugiés qui s'étaient engagés dans la Légion étrangère et les Régiments de marche de volontaires étrangers. Lors de l'offensive des troupes allemandes contre l'armée française en mai 1940, nombre de CTE qui avaient été déployées dans la zone des armées pour y réaliser des travaux de fortification et de défense passive, se retrouvèrent soudain au milieu des combats ou sous le feu de l'aviation ennemie ${ }^{57}$. Pendant la bataille de Dunkerque, par exemple, un commandant d'une compagnie stationnée dans le secteur des Flandres racontait que son unité avait été « rejetée de cantonnement en cantonnement, au milieu d'éléments de troupes, au contact avec l'ennemi " et qu'elle avait "subi des pertes sensibles ", notamment au cours du bombardement du 22 mai 1940 à Loon-Plage dans le Nord ${ }^{58}$. Certains travailleurs perdirent la vie, d'autres furent capturés par la Wehrmacht, et les plus chanceux parvinrent, tant bien que mal, à rejoindre la zone libre, après un long exode à travers la France. Parmi les 5500 Espagnols décédés au

54. P. Gaida, Camps de travail sous Vichy, op. cit., p. 23.

55. G. Dreyfus-Armand, L'exil des républicains espagnols en France, op. cit., p. 113.

56. En mai 1940, environ 100 CTE étaient affectées aux armées: Région 1 (8 CTE), Région 2 (3 CTE), Région 4 (2 CTE), Région 5 (20 CTE), Région 6 (12 CTE), Région 7 (2 CTE), Région 8 (2 CTE), Région 9 (5 CTE), Région 14 (32 CTE), Région 15 (11 CTE), Région 16 (12 CTE) et Région 17 (1 CTE).

57. D. Wingeate Pike, Españoles en el Holocausto. Vida y muerte de los republicanos en Mauthausen, Barcelone, Random House Mondadori, 2004.

58. P. Gaida, Camps de travail sous Vichy, op. cit., p. 29. 
cours de la bataille de France et de Narvik, figuraient, sans que l'on puisse en évaluer le nombre exact, des réfugiés incorporés dans les CTE. Après la défaite, la Wermacht et les Einsatzkommandos firent prisonniers plusieurs milliers de prestataires en zone occupée. Sur ordre d'Hitler, ceux-ci furent, à partir du mois de septembre 1940, dépossédés du statut de prisonniers de guerre, remis à la police secrète d'État et conduits dans des camps de concentration en Allemagne :

Ces transferts vers de camps de concentration correspondent vraisemblablement à des représailles générales décidées au plus haut niveau, à Berlin même, contre tous les anciens " combattants rouges d'Espagne ", qu'ils fassent partie du Reich ou qu' ils soient arrêtés dans les pays annexés ou occupés. L'ordre écrit daté du 25 septembre 1940 que nous avons pu retrouver n'est peut-être pas le premier; il émane en tout cas du chef de la police de sécurité allemande. Il y est prescrit de mettre en détention, au mois pour la durée de la guerre, les combattants rouges d'Espagne arrêtés par la Wermacht ou les Einsatzkommandos et de les interroger en détail [...] Ainsi, environ 7200 républicains espagnols sont envoyés dans l'ensemble concentrationnaire de Mathausen - camp central et camps annexes comme celui de Gusen - où ils sont employés à l'exploitation des carrières de granit. Le taux de mortalité des détenus marqués d'un triangle bleu "Rotspanier ", est bien supérieur à celui de l'ensemble des autres détenus du camp ${ }^{59}$.

Ce système de travail obligatoire légalisé par le décret-loi du 12 avril 1939 s'exerça au détriment des travailleurs étrangers, mais il bénéficia, en revanche, à l'État et aux employeurs privés qui surent habilement tirer leur épingle du jeu. En effet, même si la mise en place des CTE occasionna des coûts supplémentaires estimés à environ 300 millions de francs, pour l'habillement, le couchage, l'alimentation, l'achat de baraques, le salaire du personnel d'encadrement et la solde des prestataires, l'État réalisa dans le même temps des économies considérables, de l'ordre de 350 millions de francs sur les travaux de fortification. Cela n'a rien d'étonnant, car la main-d'œuvre étrangère revenait en moyenne $66 \%$ moins chère que la main-d'œuvre française.

59. G. Dreyfus-Armand, L'exil des républicains espagnols en France, op. cit., p. 123. 